\title{
Effect of Diet on Serum Creatinine in Healthy Subjects During a Phase I Study
}

\author{
Eduardo Pimenta ${ }^{\mathrm{a}, \mathrm{e}}$, Markus Jensen ${ }^{\mathrm{b}}$, David Jung ${ }^{\mathrm{b}}$, Frank Schaumann ${ }^{\mathrm{c}}$, Stefanie Boxnick ${ }^{\mathrm{c}}$, \\ Hubert Truebel ${ }^{\mathrm{a}, \mathrm{d}}$
}

\begin{abstract}
Creatinine is widely used as an indirect marker of renal function. However, interfering factors such as diet, exercise and problems with the assay can generate false results and misinterpretation of real kidney function. In this article, we report the dietary effects on serum creatinine during a phase I single dose escalation study and discuss the reasons why serum creatinine should be measured under fasting conditions.
\end{abstract}

Keywords: Kidney; Creatinine; Chronic kidney disease

\section{Introduction}

Kidney function parameters are routinely assessed in clinical studies with healthy subjects and patients during drug exposure as a safety measure to detect toxic or pharmacodynamic effects [1-3]. Creatinine is a breakdown product of creatine phosphate in muscle and its production is proportional to muscle mass. Majority of creatinine (85-90\%) is filtered by the glomerulus, but it is secreted by proximal tubular cells as well. Daily variation of creatinine production is minimal as muscle mass is relatively constant from day to day such as renal excretion of creatinine is stable under steady-state conditions. Serum creatinine is easily measured by commercially available assays, such as Jaffe reaction and is widely used as an indirect marker of renal function. However, factors have

Manuscript accepted for publication September 15, 2016

aExperimental Medicine CV/Hem, Clinical Sciences, Drug Discovery, Bayer Pharma AG, Wuppertal, Germany

${ }^{b}$ Clinical Pharmacology CV/Hem, Clinical Sciences, Drug Discovery, Bayer Pharma AG, Wuppertal, Germany

${ }^{\mathrm{c} C R S}$ Clinical Research Services Wuppertal GmbH, Wuppertal, Germany

${ }^{\mathrm{d}}$ Witten/Herdecke University, Witten, Germany

${ }^{e}$ Corresponding Author: Eduardo Pimenta, Experimental Medicine CV/Hem, Clinical Sciences, Drug Discovery, Bayer Pharma AG, Aprather Weg 18a Building 429, 42113, Wuppertal, Germany.

Email: eduardo.pimenta@bayer.com

doi: http://dx.doi.org/10.14740/jocmr2738w been described leading to falsely increased serum creatinine values which can significantly interfere with the interpretation of data in clinical trials or affect management of patients. In this article, we report an unexpected situation of systematic serum creatinine elevation during a phase I study in healthy volunteers.

\section{Case Report}

Male healthy volunteers ( $\mathrm{n}=15$ per dose step), 18 - 45 years of age, participated in a phase I, single dose escalation, singleblind, placebo-controlled, group-comparison study designed to investigate safety, tolerability, and pharmacokinetics of a novel dual acting V1a/V2 vasopressin receptor antagonist, BR-6819 (EudraCT number 2015-002254-12). This study did not enroll female subjects, because during the early stages of clinical development, knowledge of the new drug is limited and possible effects on reproduction have not yet been investigated in animal studies. Informed consent was obtained from all participants. Subjects were randomized to receive BR-6819 $1 \mathrm{mg}(\mathrm{n}=10)$, tolvaptan $30 \mathrm{mg}(\mathrm{n}=3)$ or placebo $(\mathrm{n}=2)$. One subject randomized to receive BR-6819 dropped out in dose steps 1 and 2, resulting in a total of 14 subjects completing each of these dose steps. Treatment was administered at 8:00 in the morning. Safety blood samples were collected on predosing day at $-24,-22,-20,-18,-16,-12$, and $0,+2,+4,+6,+8$, $+12,+24,+36,+48,+72$ and $+144 \mathrm{~h}$ relative to drug administration, where zero was the time of drug administration. Lunch with roasted turkey was served at -20 and $+4 \mathrm{~h}$. Unexpectedly serum creatinine (Jaffe method, Roche/Hitachi ${ }^{\circledR}$ ) significantly increased from $0.9 \pm 0.1 \mathrm{mg} / \mathrm{dL}$ (average \pm standard deviation) at baseline on the pre-dosing day $(-24 \mathrm{~h})$ to $1.3 \pm 0.3 \mathrm{mg} / \mathrm{dL}$ at $-18 \mathrm{~h}(\mathrm{P}<0.0001)$ and to $1.3 \pm 0.2 \mathrm{mg} / \mathrm{dL}$ at $+6 \mathrm{~h}$ on the dosing day $(\mathrm{P}<0.0001$, two-sided $t$-test with alpha $=0.05)$ (Fig. 1). Serum creatinine levels returned to normal levels in all subjects between 0 and $+4 \mathrm{~h}$ and after $+48 \mathrm{~h}$. There were no significant changes in other parameters such as urea, potassium or creatine kinase. Subjects tolerated the treatment with study drug well and no serious adverse events were recorded. Within the safety assessment meeting, a possible food effect was discussed as an explanation for this unexpected and obviously not drug-related increase in serum creatinine on the predosing and dosing day. 


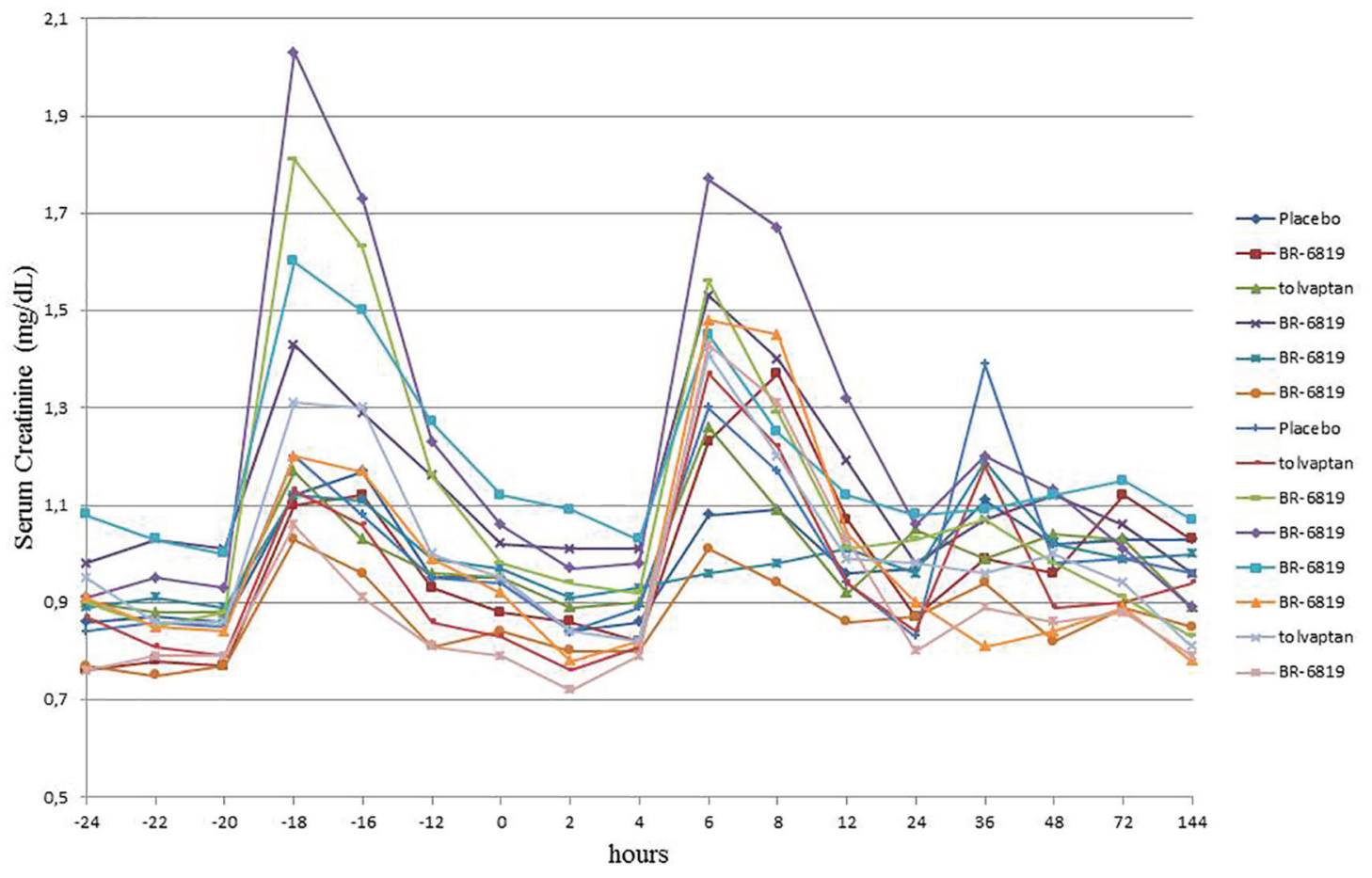

Figure 1. Serum creatinine in healthy volunteers in the pre-dosing and dosing day during dose step 1 (meals with cooked meat). Time zero corresponds to ingestion of treatment around 8:00 in the morning.

From dose step 2 onwards, the investigators decided to replace the roast turkey lunch with a vegetarian diet on predosing and dosing day. During dose step 2, serum creatinine levels showed no increase and remained stable despite the fact that the treatment with BR-6819 was escalated to $2 \mathrm{mg}$ (Fig. 2). All subsequent dose steps were performed with vegetarian diet.

\section{Discussion}

Since a similar creatinine peak was visible on both days, with and without study drug administration, drug-related toxicity was ruled out as a reason for creatinine increase. Three potential causes for falsely elevated creatinine were discussed: 1) effect of exercise; 2) problems with the laboratory assay; and 3) amount of creatinine in the diet. Physical exercise was unlikely a cause as subjects were hospitalized and under surveillance, so that a deviation from the obligation to refrain from physical activities would have been detected. Apart from this, peaks in serum creatinine occurred in all participants, in all three treatment arms and both on pre-dosing and dosing days, which does not fit to the pattern in laboratory values expected in case single subjects deviate from the resting rules as advised by protocol.

As an alternative explanation, an assay problem was discussed as it is known that some substances, such as cephalosporins can interfere with the colorimetric Jaffe reaction that was used in the laboratory [4]. However, this possibility was also an unlikely explanation, because regular use of medicines or use of any drug within 14 days before treatment with the investigational medicinal product was forbidden by protocol. A deviation from this rule would also not explain an observable creatinine increase in all trial subjects.

In order to control for a possible dietary effect, the meat containing type of meal was replaced by a vegetarian diet for subsequent dose steps, which resolved the issue. Under vegetarian diet, no unusual increase in creatinine was seen and the values measured were within normal range (Fig. 2).

In this phase I study, same procedures were performed on the pre-dosing and dosing day and subjects received exactly the same diet in order to minimize confounding factors. The diet was planned by a nutritionist and contained about $200 \mathrm{~g}$ of cooked meat per person per day. The heat from cooking converted creatine from skeletal muscle to creatinine and, therefore, caused transient increases in serum creatinine [5-7]. The variation in the elevation of serum creatinine in the current report may be explained by the fact that each four subjects could serve themselves from one serving dish, allowing them to eat unequal amounts of meat.

To the best of our knowledge, this is the first study to report transient increases in serum creatinine during a phase I clinical trial in healthy volunteers. Jacobsen et al evaluated the effect of different diets in postprandial serum creatinine in six healthy young medical students [5]. Serum creatinine significantly increased $3 \mathrm{~h}$ after ingestion of a meal of $500 \mathrm{~g}$ of goulash containing 250 - $300 \mathrm{~g}$ of beef. However, no significant increases in serum creatinine were seen after an ordinary hospital meal without meat or after ingestion of $300 \mathrm{~g}$ of raw beef. In the same study, investigators also measured concentra- 


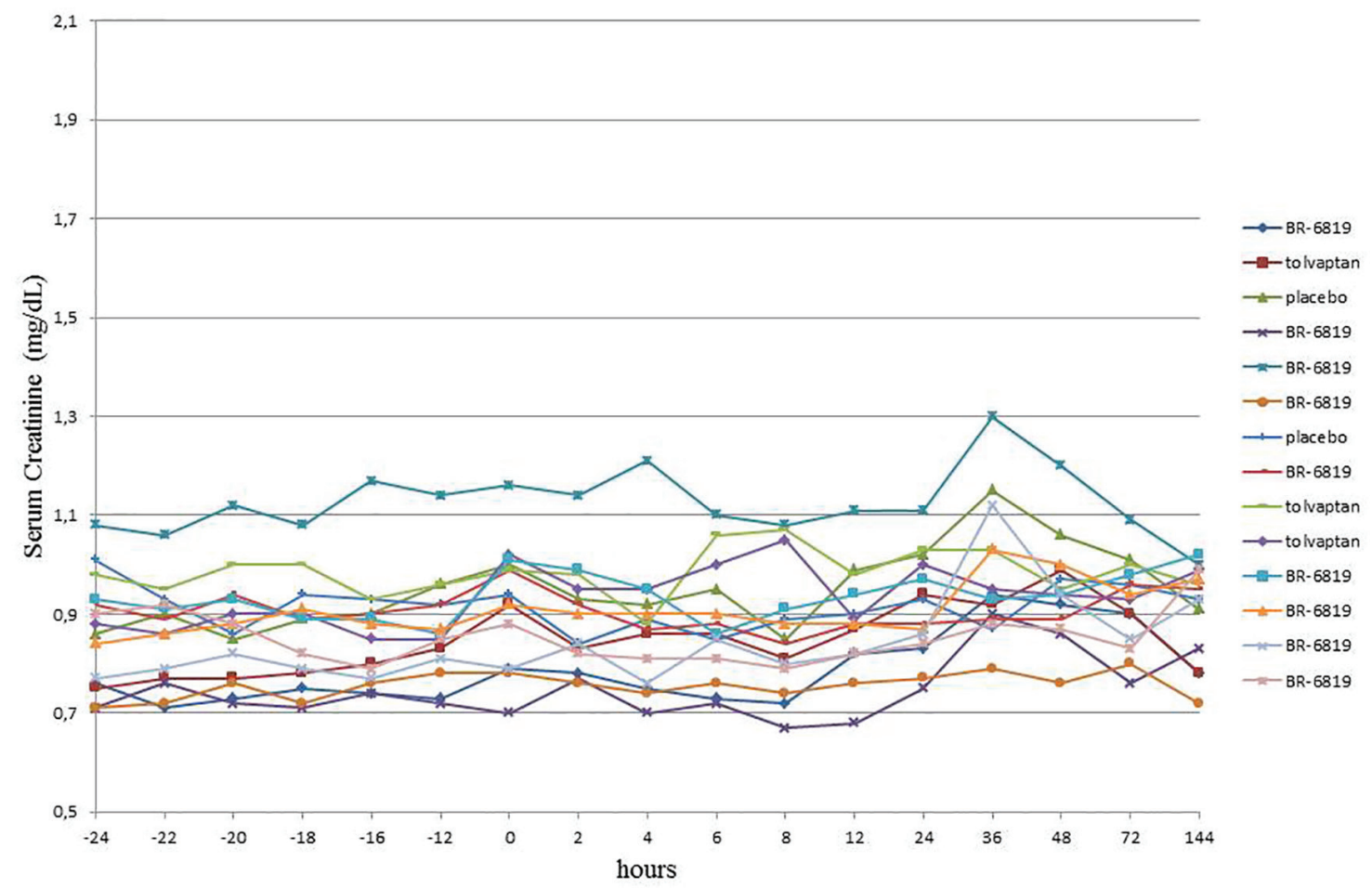

Figure 2. Serum creatinine in healthy volunteers in the pre-dosing and dosing day during dose step 2 (vegetarian meals). Time zero corresponds to ingestion of treatment around 8:00 in the morning.

tions of creatine and creatinine in the water after boiling $42 \mathrm{~g}$ of minced beef. Creatine concentrations decreased and creatinine concentrations increased in the supernatant proofing the transformation of creatine into creatinine by cooking the meat.

One can argue the meat intake in our phase I study and the studies mentioned above are well above general dietary recommendations. The dietary guidelines of the US Department of Health and Human Services and US Department of Agriculture recommend each American to consume $105 \mathrm{~g}$ of meat, poultry or eggs per day. However, average intakes of meats, poultry, and eggs are specifically higher (up to $182 \mathrm{~g}$ /day) for teen boys and adult men which matches the population in this study [8]. In Germany, the average per capita consumption of meat products in 2009 was $88.2 \mathrm{~kg}$ or $241 \mathrm{~g} /$ day [9].

Transient effects in serum creatinine are clinically relevant when estimated glomerular filtration rate (eGFR) is used to classify the stage of chronic kidney disease (CKD). In a prospective study variation in creatinine and eGFR were studied in healthy volunteers and diabetic patients with CKD after consuming meals with and without cooked meat [7]. Serum creatinine and eGFR significantly changed in healthy volunteers and patients with CKD. Six of 16 patients with CKD 3a were misclassified as CKD $3 \mathrm{~b}$ after consumption of cooked meat providing evidence that estimation of eGFR in a nonfasting condition can mistakenly affect management of patients with CKD.

Estimation of renal function in a non-fasting condition can have medical and legal implications. In healthy subjects, misdiagnosis of kidney impairment can lead to starting medi- cations or requesting additional investigations which are not necessary and can cause adverse events, complications and increased medical costs. In patients with $\mathrm{CKD}$, misclassification can also cause withdraw of life saving medications.

Our study used a strategy of repeated creatinine measurement along the day, which is, to our awareness, not the case in most phase I studies. Creatinine is usually measured less often and only in the morning. So peaks of elevated creatinine may occur frequently in clinical studies but were not detected because of the transient nature of these events.

In conclusion, ingestion of cooked meat can cause transient increases in serum creatinine to levels that are interpreted as pathologic and can lead to misinterpretation. This can potentially lead to inadequate decision making and medical management of both healthy subjects and patients with CKD. Guidelines should highlight the necessity to measure creatinine in fasting conditions.

\section{Disclosures}

All authors were involved in the design or analysis and interpretation of data. EP, MJ, DJ and HT are full time employees of Bayer Pharma AG, Germany. FS and SB are full time employees of CRS Clinical Research Services Wuppertal GmbH, Wuppertal, Germany. The study was funded by Bayer Pharma AG. This article reflects the views of the author and should not be construed to represent Bayer Pharma views or policies of its affiliates or its employees. 


\section{References}

1. Chapter 1: Definition and classification of CKD. Kidney Int Suppl (2011). 2013;3(1):19-62.

2. Fliser D, Laville M, Covic A, Fouque D, Vanholder R, Juillard L, Van Biesen W. A European Renal Best Practice (ERBP) position statement on the Kidney Disease Improving Global Outcomes (KDIGO) clinical practice guidelines on acute kidney injury: part 1: definitions, conservative management and contrast-induced nephropathy. Nephrol Dial Transplant. 2012;27(12):4263-4272.

3. Stevens LA, Coresh J, Greene T, Levey AS. Assessing kidney function - measured and estimated glomerular filtration rate. N Engl J Med. 2006;354(23):2473-2483.

4. Swain RR, Briggs SL. Positive interference with the Jaffe reaction by cephalosporin antibiotics. Clin Chem.
1977;23(7):1340-1342.

5. Jacobsen FK, Christensen CK, Mogensen CE, Andreasen F, Heilskov NS. Postprandial serum creatinine increase in normal subjects after eating cooked meat. Proc Eur Dial Transplant Assoc. 1979;16:506-512.

6. Samra M, Abcar AC. False estimates of elevated creatinine. Perm J. 2012;16(2):51-52.

7. Nair S, O'Brien SV, Hayden K, Pandya B, Lisboa PJ, Hardy KJ, Wilding JP. Effect of a cooked meat meal on serum creatinine and estimated glomerular filtration rate in diabetes-related kidney disease. Diabetes Care. 2014;37(2):483-487.

8. 2015-2020 Dietary Guidelines for Americans, 2015.

9. BMELV (Bundesministerium fur Ernahrung LuF). Statistisches Jahrbuch uber Ernahrung, Landwirtschaft und Forsten 2010. Bremerhaven: 2011. 